\title{
Detection of optical signal in the multispectral optoelectronic sensor employing cavity enhanced absorption spectroscopy
}

Jacek Wojtas, Robert Medrzycki

Institute of Optoelectronics, Military University of Technology

2 Kaliskiego Str., 00-908 Warsaw, Poland

\section{Introduction}

Cavity enhanced absorption spectroscopy was proposed in 1998 by Engel [1]. It is one of the modifications of cavity ring down spectroscopy (CRDS). The main difference between them is alignment radiation source and optical cavity. CEAS is based on injection a beam of radiation under the very small angle in relation to the optical axis of cavity (i.e. off axis). The radiation is multiple reflected inside the optical cavity. However, there is not used multipass cell like in TDLAS technique, e.g.: Herriot or White cell [2]. The optical cavity is built of two high-reflective and concave mirrors. Thus, dense structure modes are received or the modes do not occur due to overlapping. Therefore, all system has the small sensibility on the changes of a cavity and a laser frequency. Another advantage is that due to off-axis illumination of the front mirror, the source interference by the optical feedback from the cavity is eliminated. Sensors using CEAS method attain sensitivity amounting about $10^{-9} \mathrm{~cm}^{-1}[3,4]$.

Typical CEAS system designed for the measurement of a trace gases concentration is presented in Fig. 1. It consists of a pulsed radiation source, optical cavity, photoreceiver, and a signal processing system [5].

Fig. 1. Idea of CEAS setup.

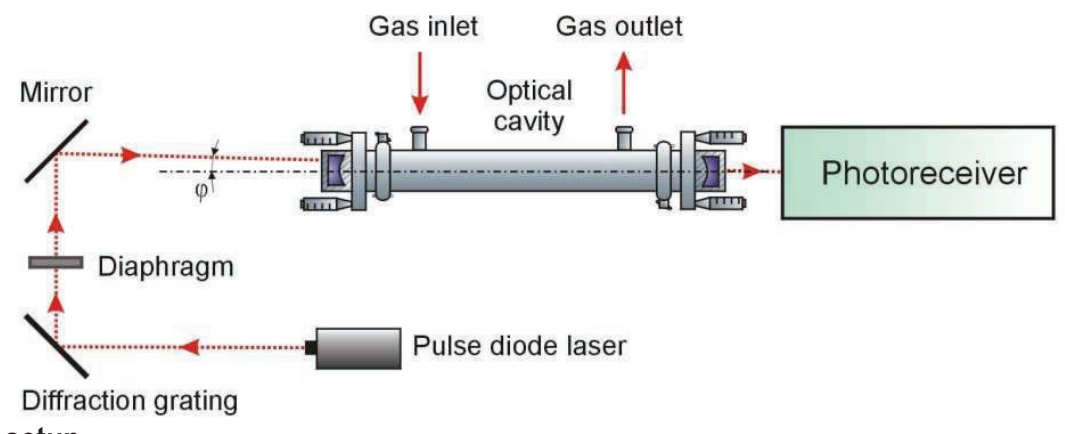

The photoreceiver registers the optical signal, the intensity I $(t)$ of which can be described as

$$
I(t)=I_{0} \cdot e^{-\frac{t}{\tau}}
$$

Moreover, the gas concentration determination is possible by two decay times measurements $(\tau$ and $\tau_{0}$ ). Firstly, $T_{0}$ is measured for the case without absorption $(\alpha=0)$. Secondly for cavity filled the analyzed gas $(\tau)$. By comparison of these two times, the absorber concentration $C$ can be achieved from

$$
C=\frac{\alpha}{\sigma}=\frac{1}{\sigma \cdot c}\left(\frac{1}{\tau}-\frac{1}{\tau_{0}}\right),
$$

where $\sigma$ denotes the absorption cross section and $c$ - light velocity.

The sensitivity of this spectroscopic method is determined with the lowest detectable concentration of analyzed gas molecules $C_{L}$, which can be described with the formula

$$
C_{L}=\frac{1}{c \cdot \sigma \cdot \tau_{0}} \cdot \delta_{\tau}=\frac{(1-R)}{\sigma \cdot L} \cdot \delta_{\tau},
$$

where $\delta_{T}$ is the relative uncertainty of the decay time measurement [6].

\section{Multispectral CEAS method idea}

In the experimental setup two lasers and two optical channels are used (Fig. 2). The setup makes it possible to detect a trace concentration of two gases with different absorption spectra at the same time. Another possibility is applying one of the channels to optical elements alignment controlling. Therefore, to register optical signal from two channels special photoreceiver was developed. There was applied special registration technique, well-known as time division multiplexing. Each of the lasers is assigned to the 
suitable measurement channel - the strictly determined temporary window. In this window, the signal from the optical cavity is registered.

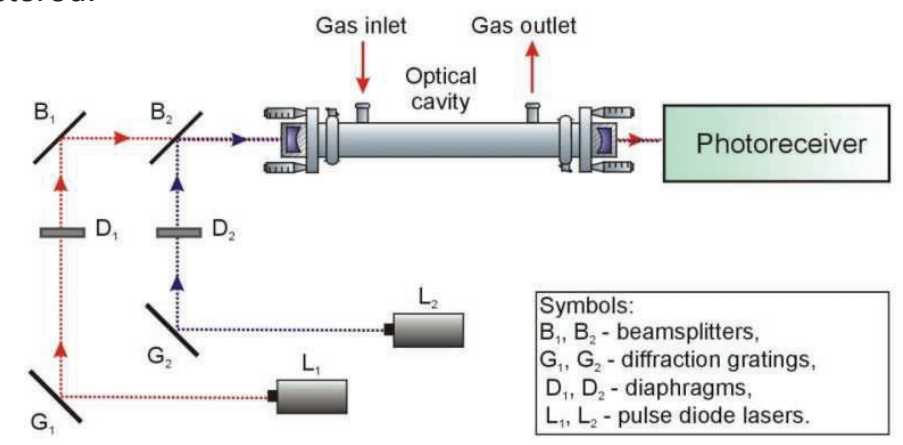

Fig. 2. Scheme of multi-spectral CEAS sensor.

As was mentioned, in the cavity without absorber the decay time $\tau_{0}$ is related to the light speed, the optical cavity length and the mirrors reflectivity. In case when the cavity is filled with the absorber the decay time $\tau$ has additionally dependent on absorption index of investigate gas. So that among at decay times the following dependence $\tau_{0}>\tau$ is observe. Thereby, a main parameter determined duration of the measuring-window is the decay time $T_{0}$ (Fig. 3).

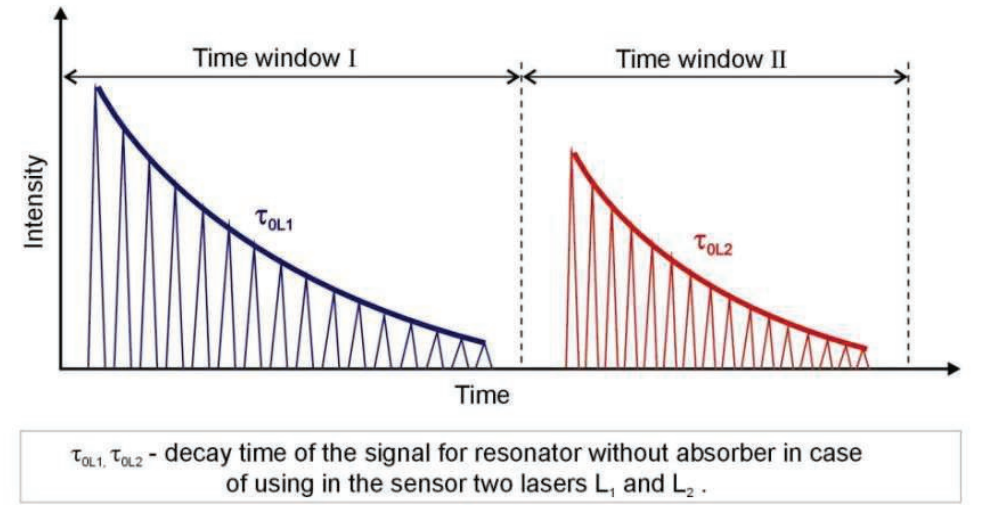

Fig. 3. Example of the output cavity signal

\section{Experimental setup}

As a radiation sources two laser diodes were applied (Fig. 4). The lasers are designed to cw mode operating at room temperatures. In the first channel, 635-nm wavelength AIGalnP laser diode was used (type DL-5038-031, Sanyo). It is usually applied as a light source in bar code readers, laser levellers and various other types of optical equipment. The main parameters of the laser are presented in Table 1.

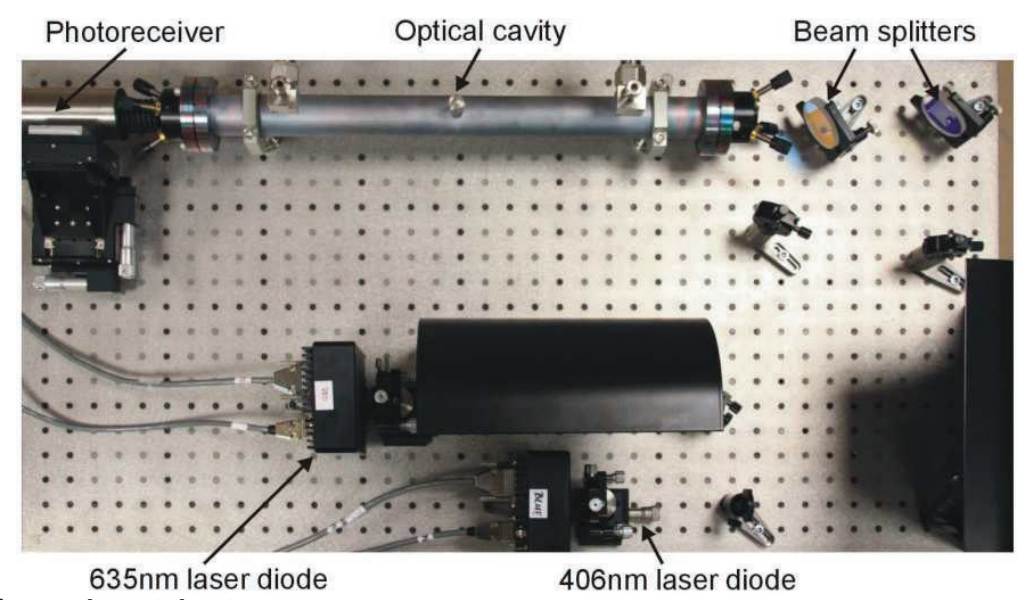

Fig. 4. Photography of experimental setup. 
Table 1. Laser diodes parameters

\begin{tabular}{|c|c|c|c|c|c|c|}
\hline $\begin{array}{c}\text { Laser } \\
\text { type }\end{array}$ & $\begin{array}{c}\text { Lasing } \\
\text { wavelength }\end{array}$ & $\begin{array}{c}\text { Threshold } \\
\text { current }\end{array}$ & $\begin{array}{c}\text { Operating } \\
\text { voltage }\end{array}$ & $\begin{array}{c}\text { Optical } \\
\text { power }\end{array}$ & $\begin{array}{c}\text { Perpendicular } \\
\text { divergence }\end{array}$ & $\begin{array}{c}\text { Parallel } \\
\text { divergence }\end{array}$ \\
\hline DL-5038-031 & $635-645 \mathrm{~nm}$ & $90-110 \mathrm{~mA}$ & $2.4-2.7 \mathrm{~V}$ & $35 \mathrm{~mW}$ & $25-35^{\circ}$ & $6-9^{\circ}$ \\
\hline GH04125A2A & $400-413 \mathrm{~nm}$ & $125-150 \mathrm{~mA}$ & $5.4-6.4 \mathrm{~V}$ & $150 \mathrm{~mW}$ & $16-25^{\circ}$ & $6-12^{\circ}$ \\
\hline
\end{tabular}

The second channel is equipped with violet laser diode (type GH04125A2A, Sharp). It is high power laser designed to sensors applications (Table 1).

Next, the laser beams were formed with diffraction gratings and diaphragms, and they were directed with beam splitters (CVI Melles Griot) into the optical cavity. The transmission characteristics of the beam splitters are presented in Fig. 5.

a)

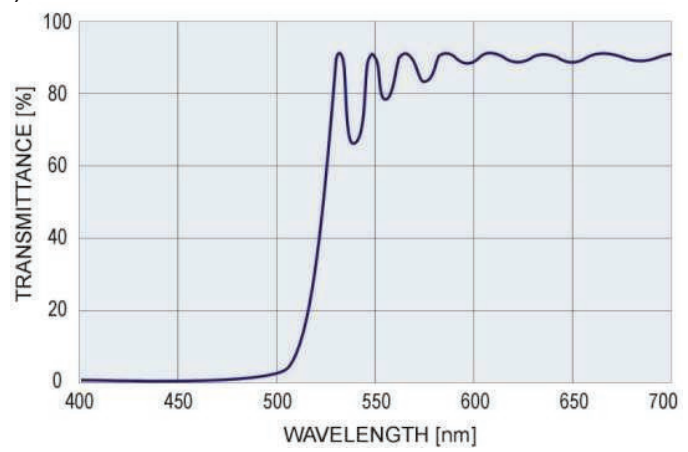

b)

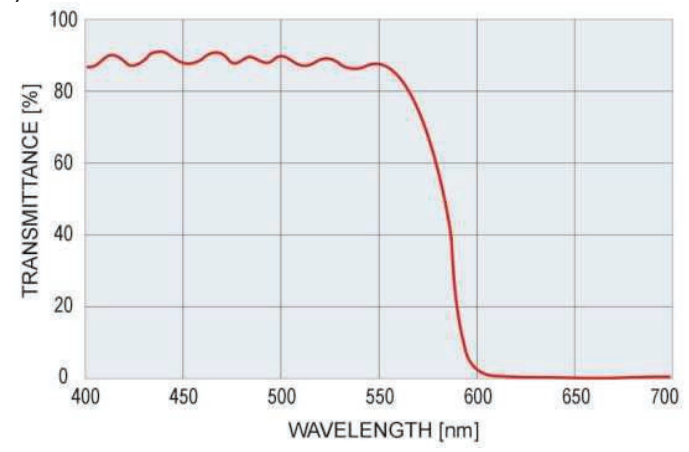

Fig. 5. Transmittance of beam splitters used in first channel (a) and in the second (b).

The optical cavity was built of two spherical mirrors, the reflectivities of which reach value of 0.999 at the wavelength of interest (Fig. 6). The distance between the mirrors was $50 \mathrm{~cm}$.

The optical signal from the cavity was registered with a photomultiplier (PMT) (type R7518, Hammamatsu). It is characterized by high gain $\left(1.1 \times 10^{7}\right)$, high speed and low dark current. Because of PMT high resistance, a transimpedance preamplifier was used to amplify signal from PMT. In the preamplifier, the operational amplifier AD 8038 type was applied. It is characterized by a wide dynamic range [7]. Next, a signal from the preamplifier was digitized with $100 \mathrm{MS} / \mathrm{s}$ sampling rate. Data from analogue-to-digital (A/D) converter were transmitted to a computer through USB interface. There was developed special software, which provides control of the measuring process and processing measuring data. The control of the measuring process embraces inspecting the data transmission, the arrangement of analogue-digital conversion parameters, archiving measuring data, and display measurement results.

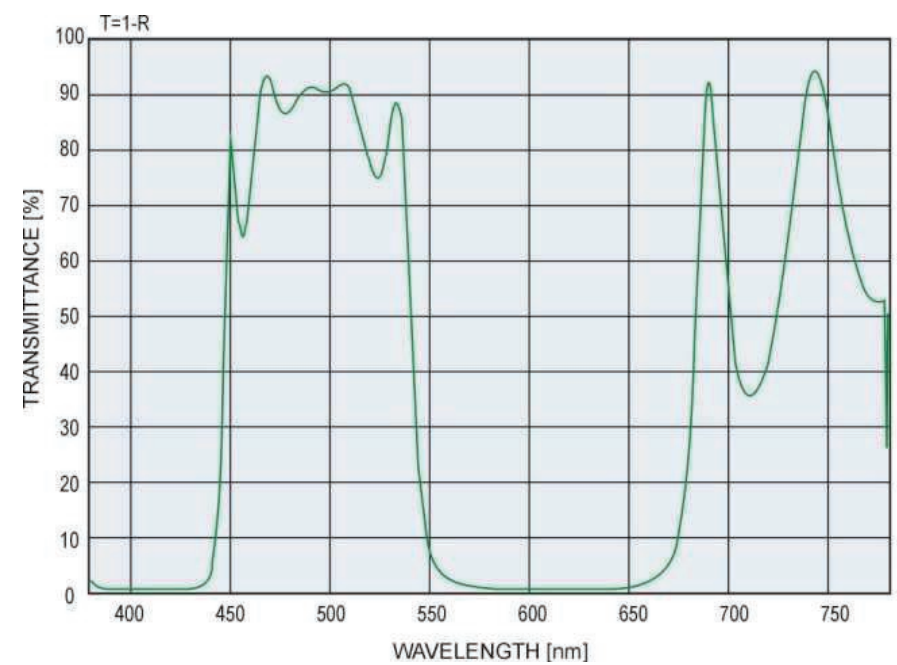

Fig. 6. Transmittance of the cavity mirrors. 
The optical signal from the cavity was registered with a photomultiplier (PMT) (type R7518, Hammamatsu). It is characterized by high gain $\left(1.1 \times 10^{7}\right)$, high speed and low dark current. Because of PMT high resistance, a transimpedance preamplifier was used to amplify signal from PMT. In the preamplifier, the operational amplifier AD 8038 type was applied. It is characterized by a wide dynamic range [7]. Next, a signal from the preamplifier was digitized with $100 \mathrm{MS} / \mathrm{s}$ sampling rate. Data from analogue-to-digital (A/D) converter were transmitted to a computer through USB interface. There was developed special software, which provides control of the measuring process and processing measuring data. The control of the measuring process embraces inspecting the data transmission, the arrangement of analogue-digital conversion parameters, archiving measuring data, and display measurement results.

\section{Optical signal registration}

According to the equation (3), the sensor sensitivity is higher when mirror reflectivity and cavity length are increased (Fig. 7). Then lower level of optical signal reaches the photodetector. Therefore, signal-to-noise ratio (SNR) is very important. Due to high value of SNR of the optical cavity, signal-to-noise ratio of an electronic circuit is the crucial parameter of CEAS sensor [8].

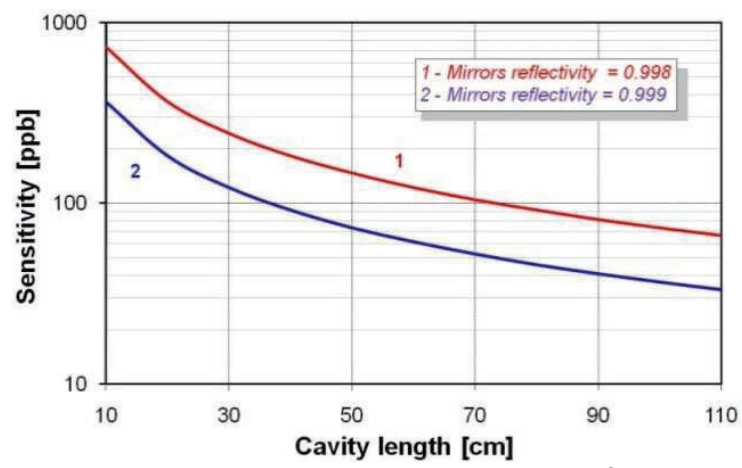

Fig. 7. Dependence of the sensor sensitivity on the cavity length $L$ and the reflectivity of mirrors $R$.

In the visible range of wavelengths, there are two types of photodetectors, which provide high sensitivity: avalanche photodiodes (APD) and photomultipliers (PMT). Currently available modules with APD include in a common housing: high voltage inverter, a temperature-compensation bias circuit, and a current-to-voltage converter. APD modules are characterized by sensitivity of about $6.3 \mathrm{pW}$ (C5460-01, Hamamatsu). However, with an increase rate of response decreases the sensitivity of photodetectors (e.g. in case C5460-01, the high band cut-off frequency of $0.1 \mathrm{MHz}$ is reported). Thus, better solution was a PMT application. Its sensitivity reaches the value of $\sim 10^{6} \mathrm{~A} / \mathrm{W}$. The other parameters of used photomultiplier were presented in Table 2.

Table 2. PMT R7518 type parameters

\begin{tabular}{|c|c|c|c|c|c|}
\hline $\begin{array}{c}\text { Spectral } \\
\text { response }\end{array}$ & $\begin{array}{c}\text { Anode sensitivity } \\
(@ 400 \mathrm{~nm})\end{array}$ & Gain & $\begin{array}{c}\text { Time } \\
\text { response }\end{array}$ & $\begin{array}{c}\text { Anode dark } \\
\text { current }\end{array}$ & $\begin{array}{c}\text { Photocathode } \\
\text { dimensions }\end{array}$ \\
\hline $185-730 \mathrm{~nm}$ & $10.2^{*} 10^{5} \mathrm{~A} / \mathrm{W}$ & $1.2^{*} 10^{7}$ & $2.2 \mathrm{~ns}$ & $0.2-2.0 \mathrm{nA}$ & $24 \times 8 \mathrm{~mm}$ \\
\hline
\end{tabular}

PMT can be treated as a current source characterized by high resistance. Therefore, to amplify signal from PMT in wide dynamic range, transimpedance preamplifier was used (Fig. 8).

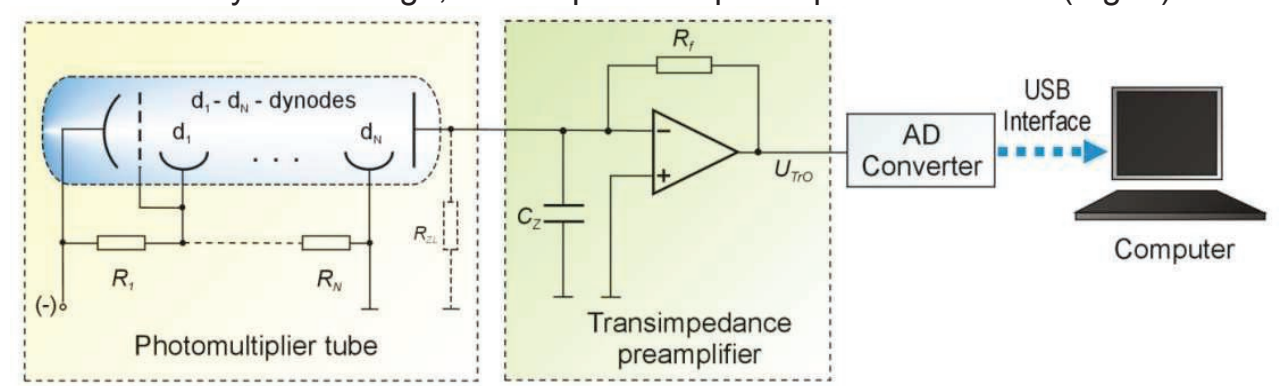

Fig. 8. Scheme of signal registration system.

Signal-to-noise ratio of the signal registration system consists of PMT, preamplifier and ADC can be described by the formula 


$$
S N R=\frac{\left(P_{s} S_{P h} G_{P h}\right)^{2} \cdot R_{f}^{2}}{\left(2 q\left(G_{P h} S_{P h} P_{s}+I_{d a}\right) \frac{\delta}{\delta-1}+\left(V_{\text {nopa }} \frac{R_{P h}+R_{f}}{R_{P h} \cdot R_{f}}\right)^{2}+I_{\text {nopa }}^{2}+\frac{4 k T_{o}}{R_{f}}\right) R_{f}^{2} \Delta f_{n}+V_{n A D C}^{2}},
$$

where $P_{S}$ - power of optical radiation, $G_{P h}$ - PMT gain, $S_{P h}$ - photocathode sensitivity, $q$ - electron charge, $\Delta f_{n}$ - noise bandwidth, $I_{d a}$ - anode dark current, $\delta$ - one stage of the PMT gain, $k$ - Boltzmann constant, $T_{0}$ - temperature, $V_{\text {nора }}, I_{\text {nора }}$ - noises of operational amplifier, $V_{\text {nADC }}$ - analogue to digital converter noise.

Analysis showed that SNR strongly depends on preamplifier parameters. There is especially important feedback resistance, $R_{f}$. This was confirmed with experimental measurements. (Fig. 9).

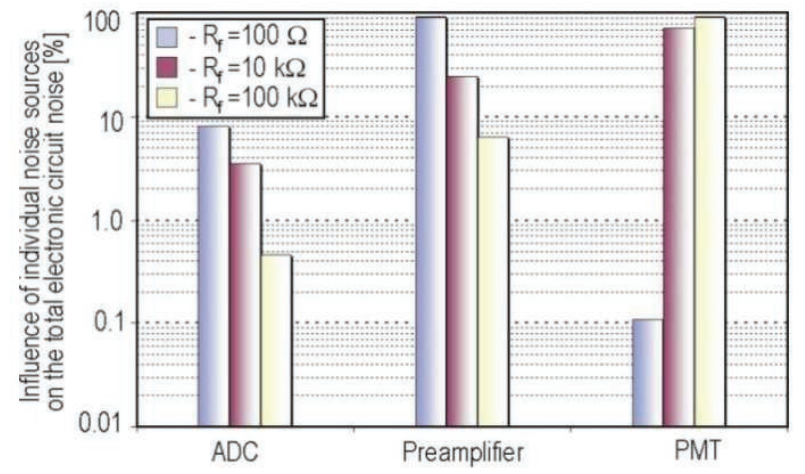

Fig. 9. Comparison of the main noise sources for different values of $R_{f}$. formula

The signal-to-noise ratio can be improved by the use of coherent averaging in compliance with

$$
S N R^{\prime}=S N R \cdot \sqrt{N_{p}},
$$

where $S N R^{\prime}$ - signal-to-noise ratio on the output A/D converter, $N_{p}$ - number of the averaging samples.

Thus, the signal-to-noise ratio is directly proportional to a root of the number of the averaging samples. Thanks to averaging the signal of over 2048 pulses, about $F=2 \%$ precision of the decay time determination was obtained. In case $\mathrm{NO}_{2}$ concentration measurements, $100 \mathrm{ppb}$ sensitivity was achieved.

\section{Summary}

In the paper, optical signal registration used in two-channel cavity enhanced absorption spectroscopy system was shown. In the experiments, the pulsed laser diodes were applied. Such solution simplifies the sensor construction. The resonator quality was determined by measuring the decay time of optical radiation from the cavity. Therefore, measurements were not sensitive to the laser power fluctuation and photodetector sensitivity fluctuation.

Analysis and measurements of SNR showed significant influence of preamplifier parameters. The best SNR was achieved when a transimpedance preamplifier was used to amplify signal from PMT. This was because of PMT high resistance. Further increase of SNR was obtained by use of coherent averaging technique. After averaging the signal of over 2048 pulses, about $2 \%$ precision of the decay time determination was obtained and sensor sensitivity of $100 \mathrm{pbb}$ for $\mathrm{NO}_{2}$ was achieved.

Moreover, developed sensor is equipped with two measurement channels. Thus it is able to detect trace concentration of two gases at the same time. For the detection of one gas, increase in sensor sensitivity can be achieved, because one gas can be investigated at the two different wavelengths of its absorption spectra. In addition, the second channel can be applied to control of the sensor optical system alignment.

The presented sensor system is of the greatest interest with respect to the possible applications. For example, it can be used in environment monitoring, luggage monitoring in ports, on airports, entry points, as well as in strategic objects and rooms also in undertakings connected with the counteraction to terrorist attacks.

\section{Acknowledgements}

This work is supported from the Ministry of Science and High Education of Poland (Project No. O N515 115836). 


\section{References}

[1] R. Engeln, G. Berden, R. Peeters, G. Meijer, Cavity enhanced absorption and cavity enhanced magnetic rotation spectroscopy, Review Of Scientific Instruments, Vol. 69, No. 11, pp. 3763 - 3769, November, 1998.

[2] D. Jean-Franqois, D. Ritz, P. Carlier, Multiple-pass cell for very-long-path infrared spectrometry, Applied Optics, Vol. 38, No. 19, pp 4145-4151, July, 1999.

[3] Nowakowski M., Wojtas J., Bielecki Z., \& Mikolajczyk J., Cavity enhanced absorption spectroscopy sensor, Acta Phys. Pol. A, 116, 363-367, 2009.

[4] Courtillot I., Morville J., Motto-Ros \& Romanini D., Sub-ppb $\mathrm{NO}_{2}$ detection by optical feedback cavityenhanced absorption spectroscopy with a blue diode laser. Appl. Phys. B, 85, pp. 407-412, 2006.

[5] J. Wojtas, A. Czyzewski, T. Stacewicz, Z. Bielecki, J.Mikolajczyk, „Cavity enhanced spectroscopy for $\mathrm{NO}_{2}$ detection", Proceedings of the SPIE, Volume 5954, pp. 174-178, 2005.

[6] Z. Bielecki, M. Leszczyński, K. Holz, L. Marona, J. Mikołajczyk, M. Nowakowski, P. Perlin, B. Rutecka, T. Stacewicz, J. Wojtas, „Sub-ppb $N O_{x}$ detection by CEAS system with blue and IR diode laser”, Computational Methods and Experimental Measurements XIV, WIT Press, pp. 809-818, 2009.

[7] Wojtas J., Czyzewski A., Stacewicz T. \& Bielecki Z., Sensitive detection of $\mathrm{NO}_{2}$ with Cavity Enhanced Spectroscopy, Optica Applicata, 36(4), pp. 461-467, 2006.

[8] A. Rogalski, Z. Bielecki, Detection of optical radiation (chapter A1.4), Handbook of optoelectronics, Taylor \& Francis, New York, London pp. 73-117, 2006.

[9] J. Wojtas, Z. Bielecki, „Signal processing system in the cavity enhanced spectroscopy”, Opto-Electron. Rev. 16(4), pp. 44-51, 2008. 